\title{
Complex Zeros of an Incomplete Riemann Zeta Function and of the Incomplete Gamma Function
}

\author{
By K. S. Kölbig
}

\begin{abstract}
Complex zeros of an incomplete Riemann zeta function and of the incomplete gamma function are calculated as functions of the upper limit $\lambda$ of the definition integrals. It becomes apparent that not all, but only some, of the zero trajectories of the incomplete Riemann zeta function join a zero of the Riemann zeta function $\zeta(s)$ for $\lambda \rightarrow \infty$. The remaining trajectories at least in the region considered, approach the zero trajectories of the incomplete gamma function.
\end{abstract}

1. Introduction. Let $s=\sigma+i t$ be a complex variable. The gamma function can then be defined for $\operatorname{Re} s=\sigma>0$ by the infinite integral

$$
\Gamma(s)=\int_{0}^{\infty} x^{s-1} e^{-x} d x .
$$

By replacing the upper limit of the integral by a parameter $\lambda>0$, one obtains the so-called incomplete gamma function [1]

$$
\gamma(s, \lambda)=P(s, \lambda) \Gamma(s)=\int_{0}^{\lambda} x^{s-1} e^{-x} d x
$$

or

$$
P(s, \lambda)=\frac{1}{\Gamma(s)} \int_{0}^{\lambda} x^{s-1} e^{-x} d x .
$$

This function plays an important role in several fields.

It seems to be much less well known that a similar procedure can be applied to the Riemann zeta function

$$
\zeta(s)=\sum_{n=1}^{\infty} \frac{1}{n^{s}}
$$

This function can be defined for $\sigma>1$ by the infinite integral

$$
\zeta(s)=\frac{1}{\Gamma(s)} \int_{0}^{\infty} \frac{x^{s-1}}{e^{x}-1} d x .
$$

Replacing the upper limit by $\lambda>0$ gives

$$
A(s, \lambda)=\frac{1}{\Gamma(s)} \int_{0}^{\lambda} \frac{x^{s-1}}{e^{x}-1} d x .^{*}
$$

We shall call this function the incomplete Riemann zeta function.

Received August 25, 1969, revised November 24, 1969.

AMS Subject Classifications. Primary 3345, 6525.

Key Words and Phrases. Incomplete Riemann zeta function, incomplete gamma function, complex zeros.

* For integers $s=p(p \geqq 2), A(p, \lambda)$ is related to the Debye functions [1].

Copyright $\odot$ 1971, American Mathematical Society 
Properties of $A(s, \lambda)$ have been investigated by Putschbach [2] in a manuscript, which, as far as the author knows, remains unpublished.

It is known that the imaginary parts $t_{m}$ of the first nontrivial zeros of the Riemann zeta function $\zeta(s)$ on the line $\sigma=\frac{1}{2}$ are given by $t_{1}=14.13473, t_{2}=21.02204$, $t_{3}=25.01086, \cdots[3]$. Since

$$
\lim _{\lambda \rightarrow \infty} A(s, \lambda)=\zeta(s),
$$

it would be interesting to know how the zeros of $A(s, \lambda)$, if there are any, approach the zeros of $\zeta(s)$, if in fact they do.

It is the aim of this paper to show, by numerical calculation, the behaviour of some of the solutions $\bar{s}(\lambda)$ of $A(\bar{s}(\lambda), \lambda)=0$ in the $s$-plane. In particular, it will be seen that not all of these functions $\bar{s}(\lambda)$ approach a nontrivial zero of $\zeta(s)$, but only some of them. The remaining curves, at least in the region considered, approach the zero trajectories $\tilde{s}(\lambda)$ of the incomplete gamma function $P(s, \lambda)$ as $\lambda \rightarrow \infty$.

2. Other Formulae for $A(s, \lambda)$ and $P(s, \lambda)$. The definitions (3) and (6) are valid only for $\sigma>0$ and $\sigma>1$, respectively. Using the series expansion for the exponential function in Eq. (3), one finds for $\sigma>0$

$$
\begin{aligned}
P(s, \lambda) & =\frac{1}{\Gamma(s)} \sum_{n=0}^{\infty} \frac{(-1)^{n}}{n !} \frac{\lambda^{s+n}}{s+n} \\
& =\frac{\lambda^{s}}{\Gamma(s)} \sum_{n=0}^{\infty} \frac{(-1)^{n}}{n !} \lambda^{n} \frac{1}{s+n} .
\end{aligned}
$$

By analytic continuation, this formula now defines $P(s, \lambda)$ for $|s|<\infty$ and $0<\lambda<\infty$, with removable singularities at $s=k(k=0,-1,-2, \cdots)$, where $P(k, \lambda)=1$. For the incomplete zeta function (6), this procedure becomes more complicated. Taking the series

$$
\frac{x}{e^{x}-1}=\sum_{n=0}^{\infty} \frac{B_{n}}{n !} x^{n},
$$

where the $B_{n}$ are the Bernoulli numbers, we see that this series converges for $|x|<2 \pi$ only. We therefore split the integration interval into two subintervals: 0 to $\lambda^{\prime}$ and $\lambda^{\prime}$ to $\lambda$, where $0<\lambda^{\prime}<2 \pi$. We substitute the series (8) into the first of these two integrals and obtain

$$
A(s, \lambda)=\frac{1}{\Gamma(s)}\left\{\sum_{n=0}^{\infty} \frac{B_{n}}{n !} \frac{\lambda^{\prime s+n-1}}{s+n-1}+\int_{\lambda}^{\lambda} \frac{x^{s-1}}{e^{x}-1} d x\right\} .
$$

For $\lambda^{\prime}=1$, this formula gives

$$
A(s, \lambda)=\frac{1}{\Gamma(s)}\left\{\sum_{n=0}^{\infty} \frac{B_{n}}{n !} \frac{1}{s+n-1}+\int_{1}^{\lambda} \frac{x^{-1}}{e^{x}-1} d x\right\}
$$

and, for $\lambda \rightarrow \infty$,

$$
\zeta(s)=\frac{1}{\Gamma(s)}\left\{\sum_{n=0}^{\infty} \frac{B_{n}}{n !} \frac{1}{s+n-1}+\int_{1}^{\infty} \frac{x^{s-1}}{e^{x}-1} d x\right\} .
$$

This last formula for $\zeta(s)$ corresponds to the decomposition of Prym for $\Gamma(s)$ [4]. 
We see here that Eqs. (9), (10) and (11) are valid also in the half plane $\sigma \leqq 1$, except at the integer points $s=1,0,-1,-2, \cdots$.

For integers $s=1-k(k \geqq 1)$ one finds from Eq. (10) using the limit

$$
\lim _{s \rightarrow 1-k}(s+k-1) \Gamma(s)=\frac{(-1)^{k-1}}{(k-1) !},
$$

and the fact that the integrals in Eqs. (10) and (11) are bounded, the result

$$
A(-k, \lambda)=\zeta(-k)=(-1)^{k} \frac{B_{k+1}}{k+1}
$$

for all $\lambda$ and $k=0,1,2, \cdots$. In particular, we have

$$
\begin{aligned}
A(0, \lambda) & =-\frac{1}{2}, \\
A(-2 k, \lambda) & =0 \quad(k \geqq 1), \\
A(-2 k+1, \lambda) & =-\frac{B_{2 k}}{2 k} \quad(k \geqq 1),
\end{aligned}
$$

and, in addition, $A(1, \lambda)=\zeta(1)=\infty$.

With the help of the expansion

$$
\frac{1}{e^{x}-1}=\sum_{n=1}^{\infty} e^{-n x} \quad(x>0)
$$

we obtain from Eq. (6)

$$
A(s, \lambda)=\frac{1}{\Gamma(s)} \sum_{n=1}^{\infty} \int_{0}^{\lambda} x^{s-1} e^{-n x} d x \quad(\sigma>1) .
$$

Making the substitution $x^{\prime}=n x$ and using Eq. (3) we have

$$
A(s, \lambda)=\sum_{n=1}^{\infty} n^{-s} P(s, n \lambda) \text {. }
$$

This relation was also found by Putschbach [2].

For the asymptotic behaviour of $P(s, \lambda)$ we find in [4] for $\operatorname{Re} s \rightarrow \infty$

$$
P(s, \lambda)=e^{s} \lambda^{s} s^{-s-1 / 2} e^{-\lambda} \frac{1}{(2 \pi)^{1 / 2}}\{1+O(1 / s)\}
$$

so that

$$
A(s, \lambda)=\frac{1}{(2 \pi)^{1 / 2}} e^{s} \lambda^{s} s^{-s-1 / 2} \sum_{n=1}^{\infty} e^{-n \lambda}\{1+O(1 / s)\} .
$$

For large, but fixed, $\lambda$ it follows for $\operatorname{Re} s \rightarrow \infty$ that

$$
A(s, \lambda) \approx P(s, \lambda) \approx \frac{1}{(2 \pi)^{1 / 2}} e^{s-\lambda} \lambda^{s} s^{-s-1 / 2} .
$$

3. Nontrivial Zeros of $\mathrm{A}(s, \lambda)$. Putschbach [2] observed that for real negative $s$ the function $A(s, \lambda)$ oscillates about the function $\zeta(s)$, and both functions have the points defined by Eq. (13) in common. Furthermore, he found that $A(s, \lambda)$ has two 


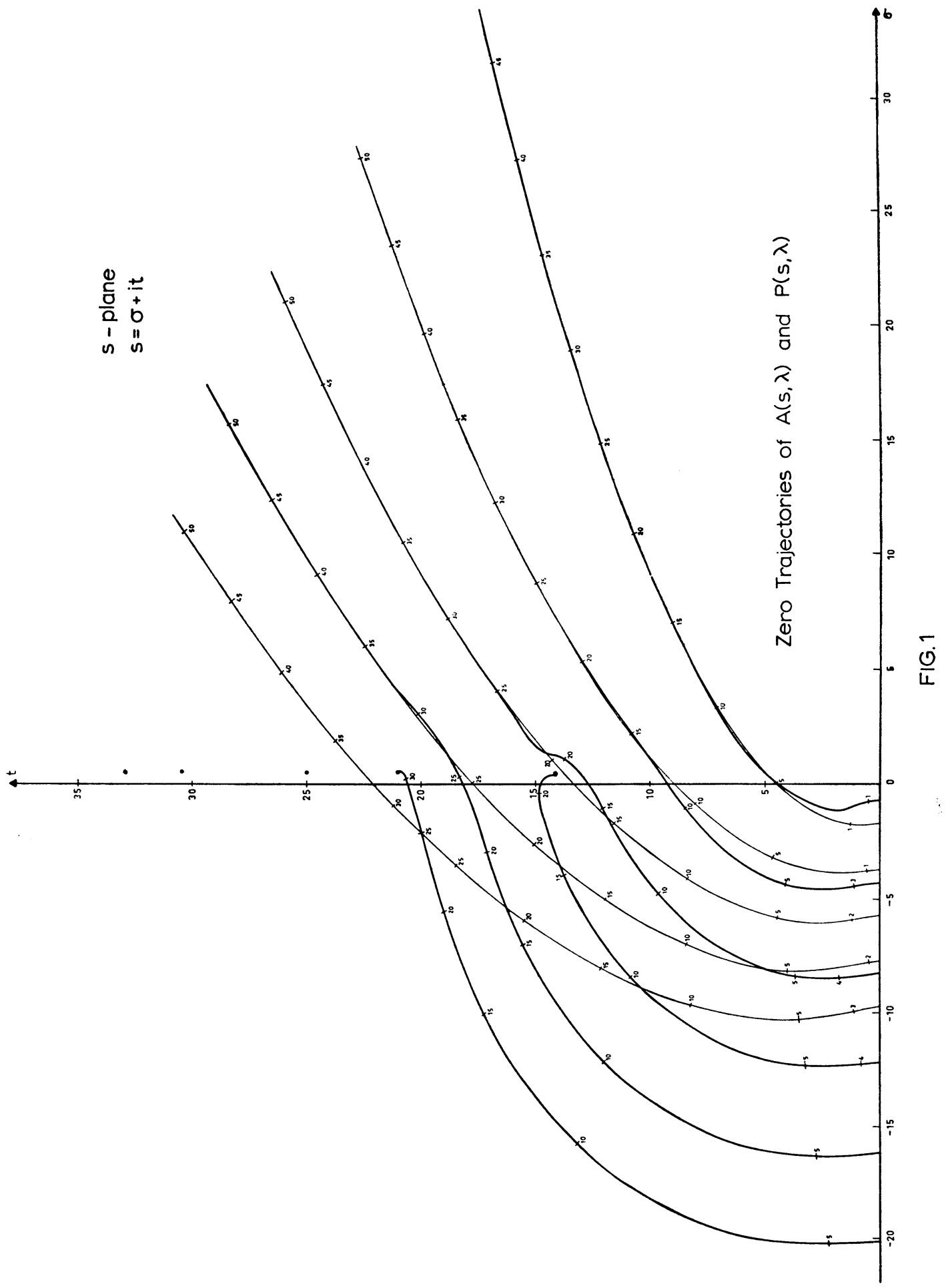


zeros in the interval $-1<s<0$, provided $\lambda \lesssim 0.6$. As $\lambda$ increases, these two zeros eventually coincide in a double zero $\bar{s}^{*}$, for $\lambda=\lambda^{*}$. For $\lambda>\lambda^{*}$, a pair of complex conjugate zeros moves into the $s$-plane. The abscissa $\bar{s}^{*}$ marks the beginning of a trajectory $\bar{s}_{1}(\lambda)$ in the half plane $t>0$, defined by $A\left(\bar{s}_{1}(\lambda), \lambda\right)=0$. Putschbach found also that a second trajectory $\bar{s}_{2}(\lambda)$ starts in the interval $-5<s<-4$. By a numerical investigation, one finds that the starting points on the negative real axis for the first six trajectories $S_{m}$ are given in Table 1 .

TABLE 1

\begin{tabular}{ccr}
\hline$m$ & $\lambda_{m}^{*}$ & $\varsigma_{m}^{*}=\bar{s}_{m}\left(\lambda_{m}^{*}\right)$ \\
\hline 1 & 0.65687 & -0.68126 \\
2 & 2.53514 & -4.29987 \\
3 & 3.35107 & -8.21375 \\
4 & 3.84904 & -12.17003 \\
5 & 4.19172 & -16.14216 \\
6 & 4.44408 & -20.12259 \\
\hline
\end{tabular}

The behaviour of $A(s, \lambda)$ near $\bar{s}_{1}^{*}$ and $\bar{s}_{2}^{*}$ is shown in Figs. M1 and M2. Figure M1 is taken from Putschbach [2]. It seems that $\tilde{s}_{m}^{*}=4-4 m-\epsilon(m)$, where $\epsilon(m)=O(1)$ for $m \rightarrow \infty$; no attempt was made to prove this relation.

The first trajectory $\bar{s}_{1}(\lambda)$ has been approximately calculated by Putschbach [2] up to $\lambda=5$. He indicated that each of the curves $\bar{s}_{m}(\lambda)$ would probably approach a nontrivial zero of $\zeta(s)$ for $\lambda \rightarrow \infty$. The author [5] extended the calculation of $\bar{s}_{1}(\lambda)$ up to $\lambda=9$. The behaviour of this trajectory in the interval $5 \leqq \lambda \leqq 9$ showed that a direct approach to the first zero of $\zeta(s)$ seemed unlikely.

A systematic investigation of the first six trajectories $\bar{s}_{m}(\lambda)$ up to $\lambda=50$ gives the result shown in Fig. 1. The first, second, third and fifth curves, $\bar{s}_{1}(\lambda), \bar{s}_{2}(\lambda), \bar{s}_{3}(\lambda)$ and $\xi_{5}(\lambda)$, do not approach a zero of $\zeta(s)$, but continue into the half plane $\sigma>1$. The fourth and the sixth curve $\bar{s}_{4}(\lambda)$ and $\bar{s}_{0}(\lambda)$, however, reach for increasing $\lambda$ the zeros $s_{1}=\frac{1}{2}+14.13473 i$ and $s_{2}=\frac{1}{2}+21.02204 i$ of $\zeta(s)$, respectively. Therefore, there is no direct correspondence between the zeros $\bar{s}_{m}(\lambda)$ of $A(s, \lambda)$ for $\lambda \rightarrow \infty$ and the zeros $s_{m}$ of $\zeta(s)$ on the line $\sigma=\frac{1}{2}$.

It is interesting to observe from Fig. 1 that the picture of the $\bar{s}_{m}(\lambda)$ curves which shows a uniform behaviour in the half plane $\sigma \lesssim-2$, becomes considerably distorted in the region around $\sigma=\frac{1}{2}$. In the half plane $\sigma \gtrsim 5$, the curves are again smooth.

The curves $\bar{s}_{4}(\lambda)$ and $\bar{s}_{6}(\lambda)$ approach the corresponding zeros $s_{1}$ and $s_{2}$ of $\zeta(s)$ in different ways, as can be seen from Fig. 1 and, in detail, from Figs. M3 and M4.

4. Zeros of $P(s, \lambda)$. An examination of the graph of the function

$$
\gamma^{*}(s, \lambda)=\lambda^{-s} P(s, \lambda)
$$




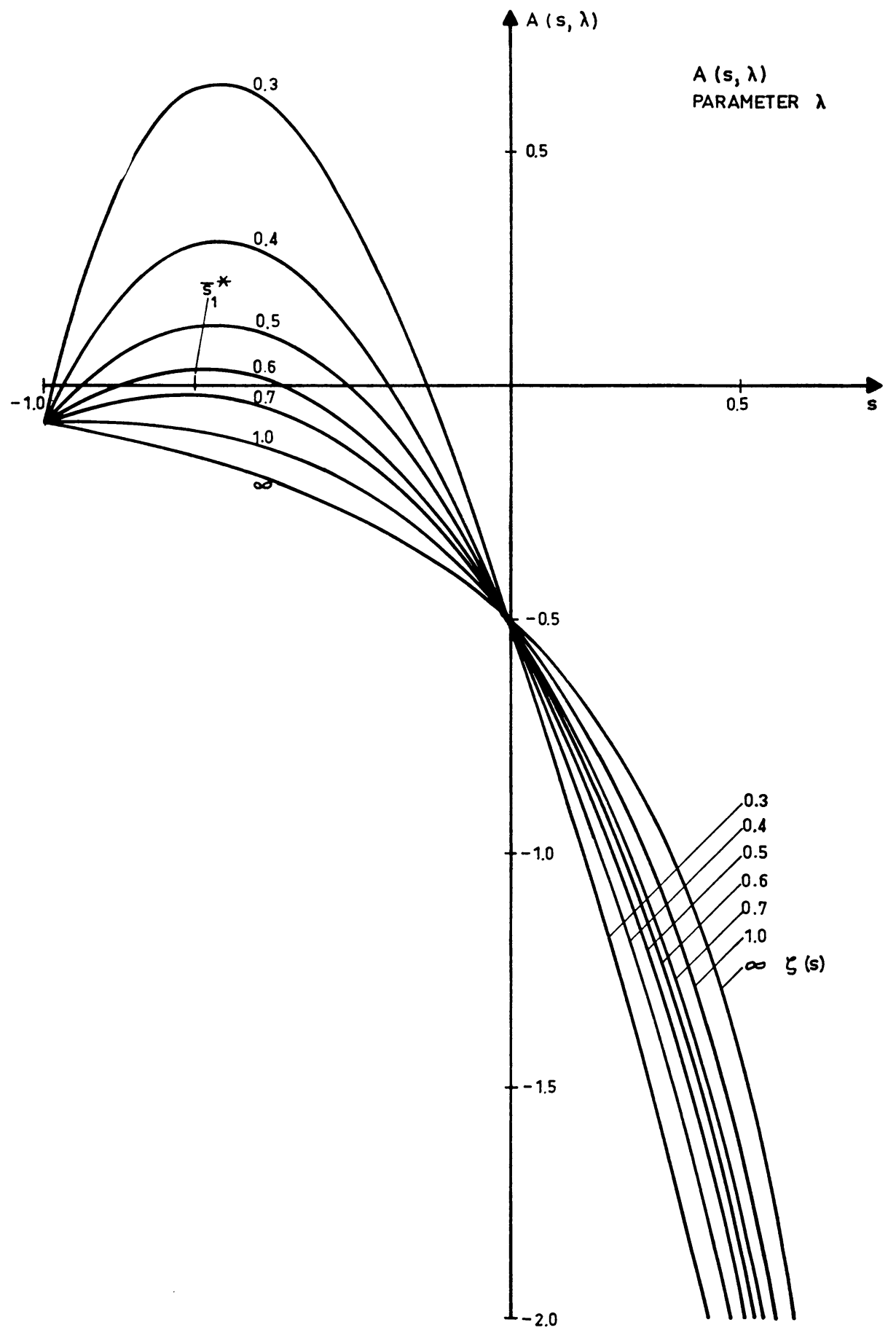

FIG. M1 
COMPLEX ZEROS

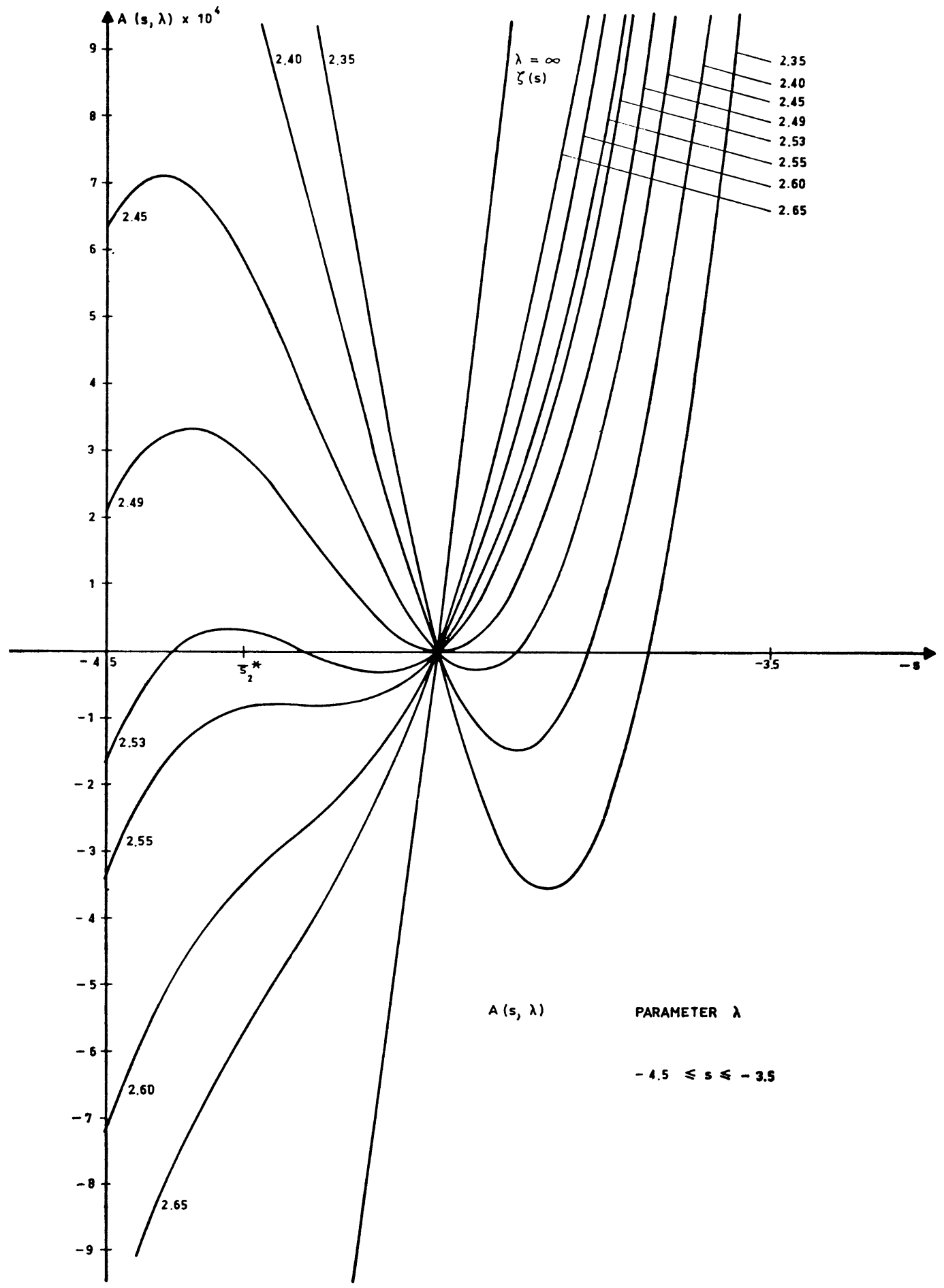

FIG.M2 


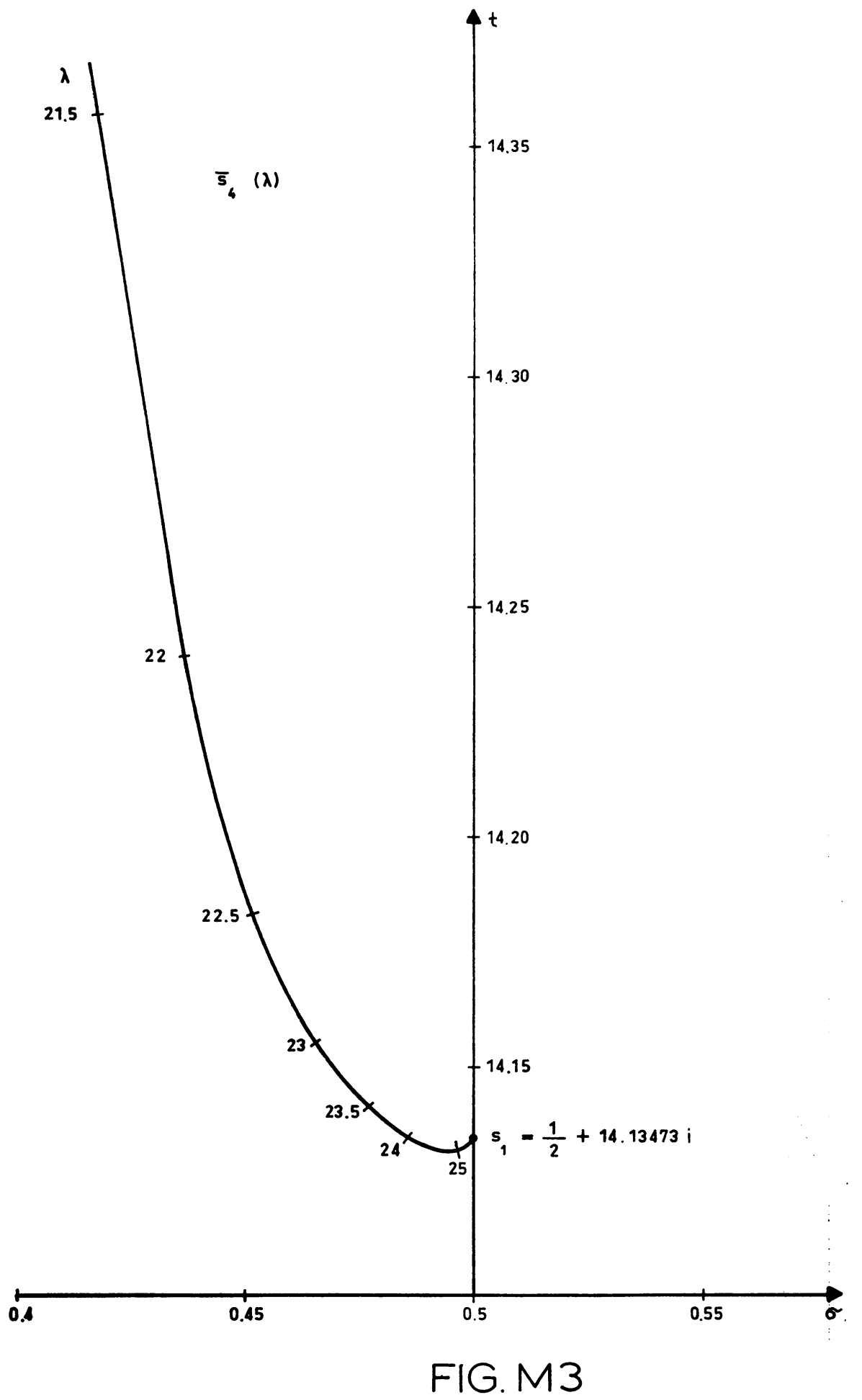




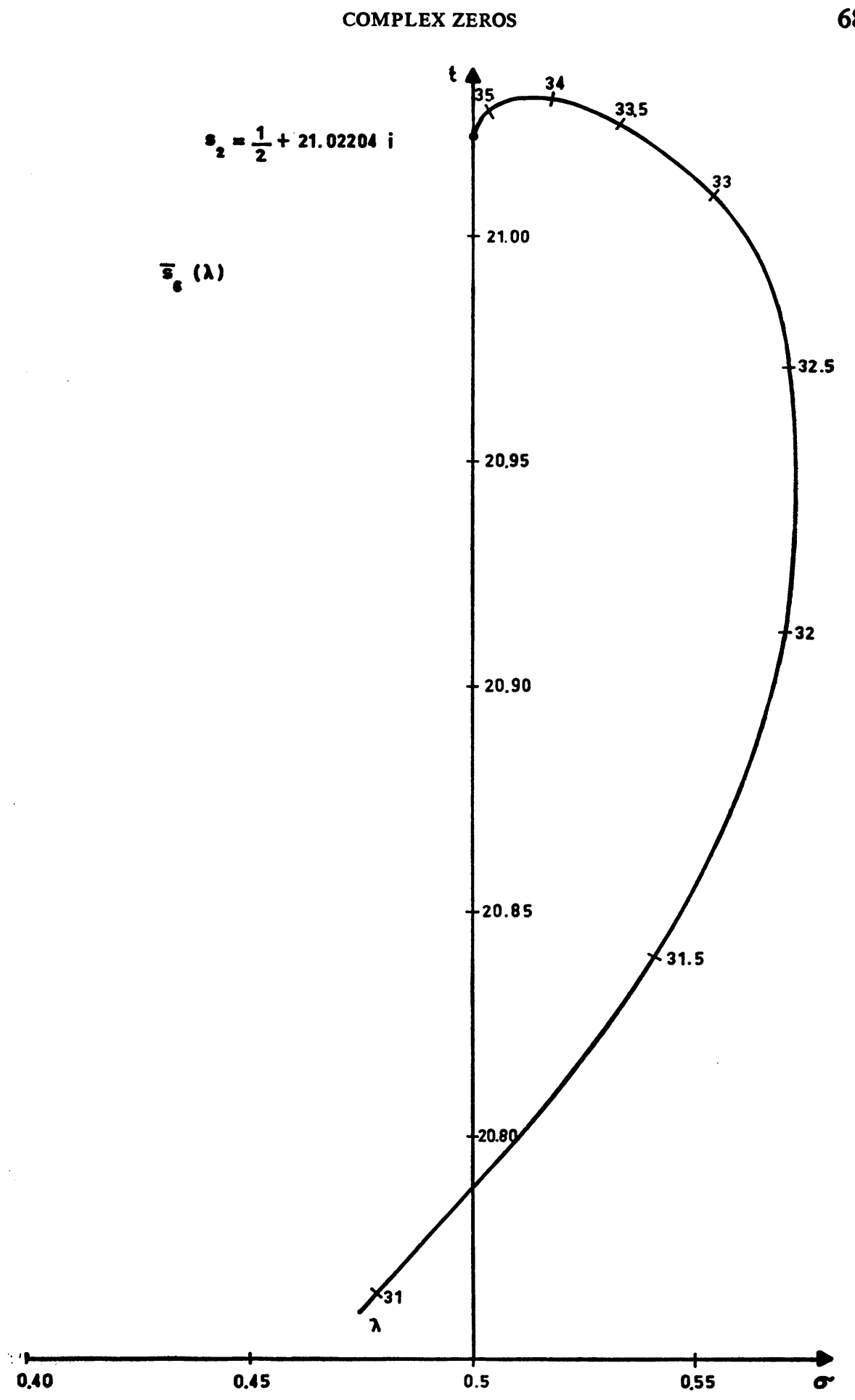

FIG. M4 
which was originally published by Tricomi [6], and which was reproduced in [1], shows that $\gamma^{*}(s, \lambda)$-and hence $P(s, \lambda)$-has double zeros in the intervals $-2<s<$ -1 and $-4<s<-3$, in both cases for $0<\lambda<1$. Furthermore, one can see from the results obtained by Tricomi [6], [7] that $P(s, \lambda)$ has a positive zero $\lambda_{10}(s)$ for each value of $s$ satisfying

$$
-2 m<s<1-2 m \quad(m=1,2,3, \cdots) .
$$

This function $\lambda_{0}(s)$ has a maximum value $\lambda_{m}^{*}$ at a certain value $s=\tilde{s}_{m}^{*}$; this means $P(s, \lambda)$ has a double zero at $\tilde{s}_{m}^{*}$ for $\lambda=\lambda_{m}^{*}$. As in the case of the incomplete zeta function, these double zeros are starting points for trajectories $\tilde{s}_{m}(\lambda)$ with $P\left(\tilde{S}_{m}(\lambda), \lambda\right)=0$ in the $s$-plane. A numerical investigation then gives the starting points for the first five trajectories $\tilde{S}_{m}(\lambda)$ as shown in Table 2 .

TABLE 2

\begin{tabular}{ccc}
\hline$m$ & $\lambda_{m}^{*}$ & $\tilde{s}_{m}^{*}=\tilde{s}_{m}\left(\lambda_{m}^{*}\right)$ \\
\hline 1 & 0.30809 & -1.64425 \\
2 & 0.77997 & -3.63887 \\
3 & 1.28634 & -5.63573 \\
4 & 1.80754 & -7.63372 \\
5 & 2.33692 & -9.63230 \\
\hline
\end{tabular}

Tricomi [6], [7] has shown that the following asymptotic expression for the positive zero $\lambda_{0}(s)$ holds for $s \rightarrow-\infty$ in the above intervals

$$
\lambda_{0}(s) \sim-\tau s-\frac{\tau}{1+\tau} \log \left[\frac{1+\tau(-\pi s / 2)^{1 / 2}}{\sin \pi s}\right] .
$$

$\tau=0.27846$ is the real root of the equation

$$
1+x+\log x=0 .
$$

A first approximation to the maximum abscissa $\tilde{S}_{m}^{*}$ can be found by differentiation of Eq. (22). This gives the following equation for $s=\tilde{s}_{m}^{*}$

$$
\frac{\tau \pi / 2}{(-2 \pi s)^{1 / 2}-\tau \pi s}+\pi \cot \pi s=1+\tau \text {. }
$$

For large $|s|$ one can neglect the first term and obtain for the remaining equation the solution

$$
\begin{aligned}
\tilde{s}_{m}^{*} & \sim 1-2 m-\frac{1}{2}-\frac{1}{\pi} \arctan \frac{1+\tau}{\pi} \\
& \sim 1-2 m-0.62302 .
\end{aligned}
$$

A comparison with Table 2 shows that for $m=5$ the error is less than $0.1 \%$. Introducing Eq. (24) into Eq. (22) gives for $m \rightarrow \infty$

$$
\lambda_{m}^{*} \sim 2 \tau m-\frac{1}{2} \frac{\tau}{1+\tau} \log m+0.03213 .
$$


The trajectories $\tilde{s}_{m}(\lambda)$ have been calculated for the given values of $m$ up to $\lambda=50$. They are shown in Fig. 1. These curves behave much more uniformly than those of the incomplete zeta function. In addition, they join, at least in the region investigated, those zero trajectories $\bar{s}_{m}(\lambda)$ of $A(s, \lambda)$ which do not end in a zero of $\zeta(s)$. This means there is a correspondence between $\bar{s}_{1}(\lambda)$ and $\tilde{s}_{1}(\lambda), \bar{s}_{2}(\lambda)$ and $\tilde{s}_{2}(\lambda), \bar{s}_{3}(\lambda)$ and $\bar{s}_{3}(\lambda)$, and between $\bar{s}_{5}(\lambda)$ and $\tilde{s}_{4}(\lambda)$.

5. The Numerical Calculation of the Zero Trajectories. The expression (7) for $P(s, \lambda)$ and the expression (10) for $A(s, \lambda)$ were used for the evaluation of $\tilde{s}_{m}(\lambda)$ and of $s_{m}(\lambda)$, respectively. Most of the calculations were carried out in double-precision arithmetic on a CDC 6600 computer, corresponding to about 28 decimal digits. For $\delta_{4}(\lambda)$ and $\tilde{s}_{5}(\lambda)$ with $\lambda>25$, it became apparent that the series (7) is no longer suitable, because of the fact that the value of $P(s, \lambda)$ lies in the round-off region. In this case, the relation

$$
P(s, \lambda)=\frac{1}{\Gamma(s)}\left\{\sum_{n=0}^{\infty} \frac{(-1)^{n}}{n !} \frac{1}{s+n}+\int_{1}^{\lambda} x^{s-1} e^{-x} d x\right\}
$$

was used.

The integrals in Eqs. (10) and (26) were calculated with an adaptive doubleprecision Gaussian integration routine [8]. The summation in Eq. (7) was carried out until the modulus of the coefficient

$$
c_{n}=\frac{(-1)^{n}}{n !} \lambda^{n}
$$

became for fixed $\lambda$ less than $10^{-30}$. The upper limit for $n$ in Eq. (10) was taken to be 60 , which is a safe value. This can be seen from the asymptotic behaviour of the Bernoulli numbers and from $B_{60} / 60 ! \approx 0.26 \times 10^{-47}$. The Bernoulli numbers were taken from [1]. The sum in Eq. (26) was calculated as far as $n=40$. In fact, the time for summing the series in Eqs. (10) or (26) is quite negligible in comparison to the time required for the computation of the integrals.

For the determination of the zeros, a library program written by Gérard [9] for solving a system of nonlinear equations by Newton's method was applied to the two equations

$$
\operatorname{Re} A(s, \lambda)=0, \quad \operatorname{Im} A(s, \lambda)=0,
$$

for finding $\bar{s}_{m}(\lambda)$, and to

$$
\operatorname{Re} P(s, \lambda)=0, \quad \operatorname{Im} P(s, \lambda)=0,
$$

for finding $\tilde{s}_{m}(\lambda)$. In both cases the unknowns were $\sigma$ and $t$. Since $\lambda^{s}$ and $1 / \Gamma(s)$ have no zeros in the region of the complex plane considered, only the moduli of these functions were taken into account (as convenient scaling factors).

In order to find the starting points $s_{m}^{*}$ and $\tilde{s}_{m}^{*}$, the systems

$$
A(s, \lambda)=0, \quad \frac{d}{d s} A(s, \lambda)=0
$$

and

$$
P(s, \lambda)=0, \quad \frac{d}{d s} P(s, \lambda)=0
$$

were solved for the unknowns $s=\sigma$ and $\lambda$, using the same program. 
TABLE M1

Zero trajectories for the incomplete zeta function $A(s, \lambda)$

\begin{tabular}{|c|c|c|c|c|c|}
\hline \multicolumn{3}{|c|}{$\bar{s}_{1}(\lambda)$} & \multicolumn{3}{|c|}{$\bar{S}_{2}(\lambda)$} \\
\hline$\lambda$ & $\sigma$ & $t$ & $\lambda$ & $\sigma$ & $t$ \\
\hline 1 & -0.78560 & 0.48873 & & & \\
\hline 2 & -1.02885 & 1.36806 & & & \\
\hline 3 & -0.99776 & 2.35217 & 3 & -4.46979 & 1.11093 \\
\hline 4 & -0.68749 & 3.31309 & 4 & -4.59882 & 2.63643 \\
\hline 5 & -0.18279 & 4.17907 & 5 & -4.35230 & 4.03199 \\
\hline 6 & 0.44072 & 4.93346 & 6 & -3.85580 & 5.24344 \\
\hline 7 & 1.12927 & 5.58612 & 7 & -3.21742 & 6.26612 \\
\hline 8 & 1.84958 & 6.15643 & 8 & -2.51077 & 7.12240 \\
\hline 9 & 2.58339 & 6.66439 & 9 & -1.78191 & 7.84322 \\
\hline 10 & 3.32208 & 7.12696 & 10 & -1.05779 & 8.45942 \\
\hline 11 & 4.06267 & 7.55706 & 11 & -0.35287 & 8.99846 \\
\hline 12 & 4.80511 & 7.96383 & 12 & 0.32647 & 9.48381 \\
\hline 13 & 5.55062 & 8.35329 & 13 & 0.97926 & 9.93510 \\
\hline 14 & 6.30061 & 8.72911 & 14 & 1.60862 & 10.36853 \\
\hline 15 & 7.05622 & 9.09343 & 15 & 2.22093 & 10.79675 \\
\hline 16 & 7.81810 & 9.44742 & 16 & 2.82516 & 11.22829 \\
\hline 17 & 8.58644 & 9.79182 & 17 & 3.43155 & 11.66670 \\
\hline 18 & 9.36111 & 10.12713 & 18 & 4.04914 & 12.11019 \\
\hline 19 & 10.14181 & 10.45382 & 19 & 4.68307 & 12.55328 \\
\hline 20 & 10.92813 & 10.77237 & 20 & 5.33384 & 12.99015 \\
\hline 21 & 11.71967 & 11.08325 & 21 & 5.99901 & 13.41705 \\
\hline 22 & 12.51604 & 11.38692 & 22 & 6.67543 & 13.83272 \\
\hline 23 & 13.31692 & 11.68382 & 23 & 7.36060 & 14.23743 \\
\hline 24 & 14.12199 & 11.97436 & 24 & 8.05285 & 14.63212 \\
\hline 25 & 14.93099 & 12.25893 & 25 & 8.75119 & 15.01776 \\
\hline 30 & 19.02744 & 13.60343 & 30 & 12.31940 & 16.83590 \\
\hline 35 & 23.19492 & 14.84000 & 35 & 15.99564 & 18.50829 \\
\hline 40 & 27.41892 & 15.99107 & 40 & 19.75974 & 20.06558 \\
\hline 45 & 31.68930 & 17.07221 & 45 & 23.59633 & 21.52848 \\
\hline 50 & 35.99862 & 18.09478 & 50 & 27.49400 & 22.91220 \\
\hline
\end{tabular}

The search for the zeros along the trajectories $\tilde{s}_{m}(\lambda)$ and $\tilde{s}_{m}(\lambda)$ was made in the following way. From an approximate value $s^{\prime}$ for a given $\lambda$ the program of Gerard calculated the true value $\bar{s}_{m}$ or $\tilde{s}_{m}$ by iteration to five decimals. The approximation $\boldsymbol{s}^{\prime}$ was found in several ways: using previous calculations [2], [5], using the initial values given in Tables 1 and 2, or, in some cases (mainly for $m \leqq 3$ and $\lambda>25$ ) by an extrapolation from the trajectories already calculated. Once the true solution $\bar{s}_{m}$ or $\tilde{s}_{m}$ had been obtained, $\lambda$ was replaced by $\lambda+\Delta \lambda$ and the solution $s(\lambda)$ was used 
TABLE M1 (cont.)

\begin{tabular}{|c|c|c|c|c|c|}
\hline \multicolumn{3}{|c|}{$\bar{S}_{3}(\lambda)$} & \multicolumn{3}{|c|}{$\bar{S}_{4}(\lambda)$} \\
\hline$\lambda$ & $\sigma$ & $t$ & $\lambda$ & $\sigma$ & $t$ \\
\hline 4 & -8.42035 & 1.74239 & 4 & -12.22581 & 0.76351 \\
\hline 5 & -8.37020 & 3.65007 & 5 & -12.35540 & 3.22021 \\
\hline 6 & -7.93471 & 5.31811 & 6 & -11.97894 & 5.34229 \\
\hline 7 & -7.26889 & 6.72371 & 7 & -11.28007 & 7.13550 \\
\hline 8 & -6.48265 & 7.88720 & 8 & -10.40516 & 8.61869 \\
\hline 9 & -5.64675 & 8.84491 & 9 & -9.44945 & 9.83465 \\
\hline 10 & -4.80426 & 9.63483 & 10 & -8.47122 & 10.83040 \\
\hline 11 & -3.98037 & 10.29071 & 11 & -7.50427 & 11.64860 \\
\hline 12 & -3.18957 & 10.84054 & 12 & -6.56699 & 12.32494 \\
\hline 13 & -2.44042 & 11.30675 & 13 & -5.66830 & 12.88786 \\
\hline 14 & -1.73880 & 11.70746 & 14 & -4.81148 & 13.35939 \\
\hline 15 & -1.09012 & 12.05809 & 15 & -3.99638 & 13.75598 \\
\hline 16 & -0.50059 & 12.37351 & 16 & -3.22067 & 14.08917 \\
\hline 17 & 0.02257 & 12.67069 & 17 & -2.48032 & 14.36568 \\
\hline 18 & 0.47204 & 12.97140 & 18 & -1.76937 & 14.58645 \\
\hline 19 & 0.84086 & 13.30522 & 19 & -1.07841 & 14.74293 \\
\hline 20 & 1.11887 & 13.72180 & 20 & -0.39060 & 14.80223 \\
\hline 21 & 1.31817 & 14.39451 & 21 & 0.29134 & 14.60119 \\
\hline 22 & 1.97996 & 15.22736 & 22 & 0.43681 & 14.23920 \\
\hline 23 & 2.69664 & 15.77953 & 23 & 0.46538 & 14.15516 \\
\hline 24 & 3.37412 & 16.26119 & 24 & 0.48555 & 14.13470 \\
\hline 25 & 4.03031 & 16.71535 & 25 & 0.49548 & 14.13199 \\
\hline 30 & 7.25345 & 18.85758 & 30 & 0.50003 & 14.13472 \\
\hline 35 & 10.57523 & 20.86303 & 35 & 0.50000 & 14.13473 \\
\hline 40 & 14.00914 & 22.73530 & 40 & 0.50000 & 14.13473 \\
\hline 45 & 15.53357 & 24.49459 & 45 & 0.50000 & 14.13473 \\
\hline 50 & 21.13411 & 26.15909 & 50 & 0.50000 & 14.13473 \\
\hline
\end{tabular}

as an initial approximation $s^{\prime}$ to $s(\lambda+\Delta \lambda)$. The increment $\Delta \lambda$ was chosen empirically to be 0.25 . In a final run, the functions $A(s, \lambda)$ and $P(s, \lambda)$ were calculated for the four arguments $s=s_{m}(\lambda) \pm 10^{-5}$ and $s=s_{m}(\lambda) \pm 10^{-5} i$. Simultaneous sign changes in the real and the imaginary parts of the functions were found in all cases.

Table M1 gives five-digit values of the zeros of $A(s, \lambda)$ for the first six trajectories $\bar{s}_{m}(\lambda)$. The values for the zeros of $P(s, \lambda)$ for the first five trajectories $\widetilde{s}_{m}(\lambda)$ are given in Table M2.

The general behaviour of the other trajectories $\bar{s}_{m}(\lambda)$ for $m>6$ and $\boldsymbol{s}_{m}(\lambda)$ for $m>5$ seems to be unknown. It is probable that $\bar{s}_{7}(\lambda)$ joins $\tilde{s}_{5}(\lambda)$ and that $\bar{s}_{8}(\lambda)$ ends in the third zero of $\zeta(s)$, that is, at $s_{3}=\frac{1}{2}+25.01086 i$. Of course, it would be of 
TABLE M1 (cont.)

\begin{tabular}{|c|c|c|c|c|c|}
\hline \multicolumn{3}{|c|}{$\bar{s}_{5}(\lambda)$} & \multicolumn{3}{|c|}{$\bar{s}_{6}(\lambda)$} \\
\hline$\lambda$ & $\sigma$ & $t$ & $\lambda$ & $\sigma$ & $t$ \\
\hline 5 & -16.32207 & 2.76585 & 5 & -20.27733 & 2.29409 \\
\hline 6 & -16.00875 & 5.34149 & 6 & -20.03007 & 5.32337 \\
\hline 7 & -15.28018 & 7.52358 & 7 & -19.27452 & 7.89489 \\
\hline 8 & -14.31946 & 9.32943 & 8 & -18.23036 & 10.02442 \\
\hline 9 & -13.24691 & 10.80833 & 9 & -17.04310 & 11.76753 \\
\hline 10 & -12.13694 & 12.01640 & 10 & -15.80341 & 13.18927 \\
\hline 11 & -11.03325 & 13.00557 & 11 & -14.56512 & 14.35053 \\
\hline 12 & -9.96006 & 13.81983 & 12 & -13.35845 & 15.30326 \\
\hline 13 & -8.92959 & 14.49492 & 13 & -12.19913 & 16.08984 \\
\hline 14 & -7.94686 & 15.05923 & 14 & -11.09411 & 16.74406 \\
\hline 15 & -7.01266 & 15.53517 & 15 & -10.04522 & 17.29263 \\
\hline 16 & -6.12530 & 15.94041 & 16 & -9.05141 & 17.75653 \\
\hline 17 & -5.28173 & 16.28904 & 17 & -8.11007 & 18.15231 \\
\hline 18 & -4.47809 & 16.59257 & 18 & -7.21791 & 18.49308 \\
\hline 19 & -3.71009 & 16.86083 & 19 & -6.37143 & 18.78927 \\
\hline 20 & -2.97327 & 17.10295 & 20 & -5.56728 & 19.04931 \\
\hline 21 & -2.26341 & 17.32832 & 21 & -4.80245 & 19.28000 \\
\hline 22 & -1.57738 & 17.54750 & 22 & -4.07449 & 19.48688 \\
\hline 23 & -0.91446 & 17.77246 & 23 & -3.38168 & 19.67441 \\
\hline 24 & -0.27737 & 18.01521 & 24 & -2.72327 & 19.84601 \\
\hline 25 & 0.32901 & 18.28536 & 25 & -2.09984 & 20.00399 \\
\hline 26 & 0.90173 & 18.58923 & 26 & -1.51390 & 20.14945 \\
\hline 27 & 1.44362 & 18.93147 & 27 & -0.97106 & 20.28241 \\
\hline 28 & 1.96507 & 19.31596 & 28 & -0.48192 & 20.40298 \\
\hline 29 & 2.48362 & 19.74262 & 29 & -0.06373 & 20.51502 \\
\hline 30 & 3.01833 & 20.20151 & 30 & 0.26240 & 20.63091 \\
\hline 35 & 5.98277 & 22.51928 & 31 & 0.47862 & 20.76608 \\
\hline 40 & 9.15331 & 24.66536 & 32 & 0.57021 & 20.91242 \\
\hline 45 & 12.42733 & 26.67402 & 33 & 0.55305 & 21.00902 \\
\hline 50 & 15.78768 & 28.57536 & 34 & 0.51789 & 21.03077 \\
\hline & & & 35 & 0.50341 & 21.02830 \\
\hline & & & 36 & 0.49979 & 21.02461 \\
\hline & & & 37 & 0.49944 & 21.02279 \\
\hline & & & 38 & 0.49968 & 21.02217 \\
\hline & & & 39 & 0.49988 & 21.02202 \\
\hline & & & 40 & 0.49996 & 21.02201 \\
\hline
\end{tabular}


TABLE M2

Zero trajectories for the incomplete gamma function $P(s, \lambda)$

\begin{tabular}{|c|c|c|c|c|c|}
\hline \multicolumn{3}{|c|}{$\tilde{s}_{1}(\lambda)$} & \multicolumn{3}{|c|}{$\tilde{s}_{2}(\lambda)$} \\
\hline$\lambda$ & $\sigma$ & $t$ & $\lambda$ & $\sigma$ & $t$ \\
\hline 1 & -1.72630 & 1.23809 & 1 & -3.72647 & 0.54067 \\
\hline 2 & -1.45710 & 2.29282 & 2 & -3.88891 & 1.83256 \\
\hline 3 & -1.01952 & 3.13777 & 3 & -3.77348 & 2.89397 \\
\hline 4 & -0.49804 & 3.86483 & 4 & -3.51978 & 3.82369 \\
\hline 5 & 0.07663 & 4.51288 & 5 & -3.18074 & 4.66264 \\
\hline 6 & 0.68922 & 5.10302 & 6 & -2.78218 & 5.43355 \\
\hline 7 & 1.33072 & 5.64833 & 7 & -2.33889 & 6.15075 \\
\hline 8 & 1.99532 & 6.15758 & 8 & -1.86027 & 6.82406 \\
\hline 9 & 2.67894 & 6.63702 & 9 & -1.35275 & 7.46057 \\
\hline 10 & 3.37863 & 7.09127 & 10 & -0.82094 & 8.06563 \\
\hline 11 & 4.09212 & 7.52390 & 11 & -0.26832 & 8.64339 \\
\hline 12 & 4.81764 & 7.93769 & 12 & 0.30244 & 9.19716 \\
\hline 13 & 5.55379 & 8.33488 & 13 & 0.88921 & 9.72965 \\
\hline 14 & 6.29942 & 8.71731 & 14 & 1.49025 & 10.24307 \\
\hline 15 & 7.05355 & 9.08649 & 15 & 2.10414 & 10.73930 \\
\hline 16 & 7.81540 & 9.44370 & 16 & 2.72968 & 11.21991 \\
\hline 17 & 8.58427 & 9.79002 & 17 & 3.36586 & 11.68626 \\
\hline 18 & 9.35957 & 10.12639 & 18 & 4.01180 & 12.13952 \\
\hline 19 & 10.14080 & 10.45361 & 19 & 4.66674 & 12.58071 \\
\hline 20 & 10.92751 & 10.77239 & 20 & 5.33003 & 13.01074 \\
\hline 21 & 11.71931 & 11.08334 & 21 & 6.00107 & 13.43039 \\
\hline 22 & 12.51585 & 11.38702 & 22 & 6.67935 & 13.84036 \\
\hline 23 & 13.31682 & 11.68390 & 23 & 7.36442 & 14.24129 \\
\hline 24 & 12.12194 & 11.97442 & 24 & 8.05585 & 14.63374 \\
\hline 25 & 14.93097 & 12.25897 & 25 & 8.75327 & 15.01822 \\
\hline 30 & 19.02744 & 13.60343 & 30 & 12.31949 & 16.83573 \\
\hline 35 & 23.19492 & 14.84000 & 35 & 15.99563 & 18.50828 \\
\hline 40 & 27.41892 & 15.99107 & 40 & 19.75974 & 20.06558 \\
\hline 45 & 31.68930 & 17.07221 & 45 & 23.59633 & 21.52848 \\
\hline 50 & 35.99862 & 18.09478 & 50 & 27.49400 & 22.91220 \\
\hline
\end{tabular}


TABLE M2 (cont.)

\begin{tabular}{|c|c|c|c|c|c|}
\hline \multicolumn{3}{|c|}{$\tilde{s}_{3}(\lambda)$} & \multicolumn{3}{|c|}{$\xi_{4}(\lambda)$} \\
\hline$\lambda$ & $\sigma$ & $t$ & $\lambda$ & $\sigma$ & $t$ \\
\hline 2 & -5.90041 & 1.19521 & 2 & -7.71370 & 0.47288 \\
\hline 3 & -6.03282 & 2.39330 & 3 & -8.04938 & 1.78032 \\
\hline 4 & -5.98035 & 3.45439 & 4 & -8.16969 & 2.93895 \\
\hline 5 & -5.81495 & 4.41961 & 5 & -8.15190 & 3.99890 \\
\hline 6 & -5.57166 & 5.31319 & 6 & -8.03936 & 4.98352 \\
\hline 7 & -5.27047 & 6.14692 & 7 & -7.85680 & 5.90778 \\
\hline 8 & -4.92399 & 6.93397 & 8 & -7.61978 & 6.78206 \\
\hline 9 & -4.54079 & 7.68074 & 9 & -7.33885 & 7.61394 \\
\hline 10 & -4.12697 & 8.39283 & 10 & -7.02150 & 8.40915 \\
\hline 11 & -3.68706 & 9.07462 & 11 & -6.67328 & 9.17221 \\
\hline 12 & -3.22455 & 9.72963 & 12 & -6.29844 & 9.90672 \\
\hline 13 & -2.74218 & 10.36072 & 13 & -5.90030 & 10.61566 \\
\hline 14 & -2.24214 & 10.97027 & 14 & -5.48153 & 11.30149 \\
\hline 15 & -1.72625 & 11.56030 & 15 & -5.04430 & 11.96630 \\
\hline 16 & -1.19602 & 12.13251 & 16 & -4.59042 & 12.61187 \\
\hline 17 & -0.65271 & 12.68839 & 17 & -4.12141 & 13.23974 \\
\hline 18 & -0.09741 & 13.22922 & 18 & -3.63854 & 13.85125 \\
\hline 19 & 0.46894 & 13.75612 & 19 & -3.14293 & 14.44758 \\
\hline 20 & 1.04552 & 14.27010 & 20 & -2.63553 & 15.02977 \\
\hline 21 & 1.63163 & 14.77202 & 21 & -2.11719 & 15.59876 \\
\hline 22 & 2.22662 & 15.26267 & 22 & -1.58864 & 16.15537 \\
\hline 23 & 2.82993 & 15.74276 & 23 & -1.05054 & 16.70036 \\
\hline 24 & 3.44107 & 16.21293 & 24 & -0.50348 & 17.23439 \\
\hline 25 & 4.05957 & 16.67374 & 25 & 0.05203 & 17.75808 \\
\hline 30 & 7.24939 & 18.85435 & 26 & 0.61550 & 18.27199 \\
\hline 35 & 10.57482 & 20.86339 & 27 & 1.18651 & 18.77664 \\
\hline 40 & 14.00916 & 22.73534 & 28 & 1.76467 & 19.27248 \\
\hline 45 & 17.53358 & 24.49459 & 29 & 2.34962 & 19.75995 \\
\hline 50 & 21.13411 & 26.15909 & 30 & 2.94103 & 20.23945 \\
\hline & & & 35 & 5.98537 & 22.52930 \\
\hline & & & 40 & 9.15462 & 24.66516 \\
\hline & & & 45 & 12.42733 & 26.67388 \\
\hline & & & 50 & 15.78767 & 28.57535 \\
\hline
\end{tabular}


COMPLEX ZEROS

TABLE M2 (cont.)

\begin{tabular}{r|r|r}
\hline \multicolumn{1}{c}{} & \multicolumn{1}{|c}{$\tilde{s}_{5}(\lambda)$} & \\
\hline$\lambda$ & $\sigma$ & $t$ \\
\hline 3 & -9.91210 & 1.10407 \\
4 & -10.18817 & 2.34009 \\
5 & -10.30294 & 3.47597 \\
6 & -10.30721 & 4.53477 \\
7 & -10.23006 & 5.53153 \\
8 & -10.08983 & 6.47674 \\
9 & -9.89889 & 7.37806 \\
10 & -9.66605 & 8.24133 \\
11 & -9.39781 & 9.07115 \\
12 & -9.09914 & 9.87121 \\
13 & -8.77394 & 10.64453 \\
14 & -8.42531 & 11.39365 \\
15 & -8.05577 & 12.12068 \\
16 & -7.66743 & 12.82746 \\
17 & -7.26204 & 13.51558 \\
18 & -6.84108 & 14.18640 \\
19 & -6.40583 & 14.84115 \\
20 & -5.95739 & 15.48090 \\
21 & -5.49672 & 16.10661 \\
22 & -5.02466 & 16.71913 \\
23 & -4.54196 & 17.31925 \\
24 & -4.04928 & 17.90766 \\
25 & -3.54722 & 18.48499 \\
26 & -3.03631 & 19.05184 \\
27 & -2.51703 & 19.60873 \\
28 & -1.98983 & 20.15614 \\
29 & -1.45510 & 20.69454 \\
30 & -0.91320 & 21.22434 \\
35 & 1.89287 & 23.75684 \\
40 & 4.83715 & 26.12205 \\
45 & 7.89594 & 28.34849 \\
50 & 11.05178 & 30.45745 \\
& & \\
\hline
\end{tabular}


interest to be able to answer the-probably difficult-question: which of the trajectories $\bar{s}_{m}(\lambda)$ end in a zero of $\zeta(s)$ and which do not?

Acknowledgements. The author's attention was drawn to this problem by the late Professor Dr. A. Walther, then at Technische Hochschule Darmstadt, Germany.

I wish to thank G. A. Erskine and G. C. Sheppey (now at Wellcome Foundation, London) for helpful and encouraging discussions.

Note added in proof. After having finished the paper, the author found that Franklin [10] has calculated the zeros $\tilde{s}_{1}(1)$ and $\tilde{s}_{2}(1)$ of $P(s, 1)$ to seven decimals. The first five decimals agree with those given in Table M2. Also, Gronwall [11] has proved that $\tilde{s}_{1}(1), \tilde{s}_{2}(1)$ and their complex conjugates are the only complex zeros of $P(s, 1)$.

CERN

Geneva, Switzerland

1. M. Abramowitz \& I. A. Stegun (Editors), Handbook of Mathematical Functions, with Formulas, Graphs and Mathematical Tables, Nat. Bur. Standards Appl. Math. Series, 55, Superintendent of Documents, U.S. Government Printing Office, Washington, D.C., 1965. MR 31 \#1400.

2. R. PutschBach, Untersuchungen über die unvollständige Riemannsche Zetafunktion, Inst. f. Praktische Mathematik (IPM), Techn. Hochschule Darmstadt, 1948?. (Unpublished.)

3. E. JAHNKE, F. EMDE \& F. Lösch, Tables of Higher Functions, McGraw-Hill, New York and Verlagsgesellschaft, Stuttgart, 1960. MR 22 \#5140.

4. F. Lösch \& F. ScHOBLIK, Die Fakultät (Gammafunktion) und verwandte Funktionen mit besonderer Berücksichtigung ihrer Anwendungen, B. G. Teubner Verlagsgesellschaft, Leipzig, 1951. MR 13, 938.

5. K. S. KöLBIG, Uber die Riemannsche Zetafunktion, Diplomarbeit, Inst. f. Praktische Mathematik (IPM), Techn. Hochschule Darmstadt, 1960. (Unpublished.)

6. F. G. TrIComI, Funzioni ipergeometriche confluenti, Edizioni Cremonese, Roma, 1954. MR 17, 967.

7. F. G. Tricomi, "Asymptotische Eigenschaften der unvollständigen Gammafunktion," Math. Z., v. 53, 1950, pp. 136-148. MR 13, 553.

8. K. S. KöLbIG, DGAUSS-Double Precision Gaussian Integration, CERN 6000 Series Program Library D109, 1968. (Unpublished.)

9. M. GÉRARD, NEWTON-Solution of $n$ Simultaneous Non-Linear Equations in $n$ Unknowns by Newton's Method, CERN 6000 Series Program Library C400, 1968. (Unpublished.)

10. P. Franklin, "Calculation of the complex zeros of the function $P(z)$ complementary to the incomplete gamma function," Ann. of Math. (2), v. 21, 1919, pp. 61-63.

11. F. H. Gronwall, "Sur les zéros des fonctions $P(z)$ et $Q(z)$ associées à la fonction gamma," Ann. Ecole Norm. Sup. (3), v. 33, 1916, pp. 381-393. 